\title{
La percepción de los alumnos sobre la adquisición de competencias del Trabajo Fin de Grado en ADE. Un análisis en la Universidad Loyola Andalucía.
}

\author{
Students' perceptions about acquisition of competencies of final project \\ in Business Administration. The case of Universidad Loyola Andalucía.
}

\author{
Mercedes Ruiz Lozano (mruiz@uloyola.es) \\ Pilar Tirado Valencia (ptirado@uloyola.es) \\ Universidad Loyola Andalucía (España) \\ http://dx.doi.org/10.12795/EDUCADE.2016.107.03
}

RESUMEN: El desarrollo y la evaluación de los Trabajos Fin de Grado (TFG) en los planes de estudio están suponiendo todo un reto para el claustro de las universidades.

La complejidad del proceso de elaboración del trabajo, la distinta casuística en cuanto a temas, competencias a evaluar y diversidad de profesorado, nos ha llevado a preocuparnos por conocer cuál es la percepción del alumno en relación a su proceso de aprendizaje y adquisición de competencias en esta materia.

Un estudio exploratorio, basado en una encuesta realizada a los alumnos de ADE que han defendido su trabajo en el curso 2014-15, en la Facultad de Ciencias Económicas y Empresariales de la Universidad Loyola Andalucía, nos ha permitido poner de manifiesto cuál es la percepción del alumno en relación a todo el proceso del TFG.

Su análisis nos ha permitido detectar que el TFG está suponiendo un retraso en la finalización de los estudios de grado, pero que existe un nivel de satisfacción alto con su desarrollo. Así mismo, es de resaltar la correlación que existe entre el interés por el tema a desarrollar y la calificación obtenida.

PALABRAS CLAVE: Trabajo fin de grado, competencias, resultados de aprendizaje, percepción

\begin{abstract}
The development and evaluation of the Final Project (FP) in the curriculum are posing a challenge for the university faculty.

The complexity of the process of making this FP, the different casuistry regarding possible topics, the different competencies to assess and teachers diversity has led us to worry about knowing what is the perception of students in relation to their learning and skills acquisition in this area.

A exploratory study based on a survey of Business Administration' students who have defended their work in the 2014-15 course at the Faculty of Economics and Business Administration at Loyola University Andalusia, has allowed us to show what is the perception of the student in relation to the whole process of the FP.

The analysis revealed that the FP is supposing a delay in the completion of undergraduate studies, but that there is also a high level of satisfaction with the development of the FP. We must also highlight the correlation between interest in the topic to develop and marks obtained in the FP.
\end{abstract}

KEYWORDS: Final Project, competencies, learning outcomes, perception 


\section{INTRODUCCIÓN}

El Real Decreto 1393/2007, de 29 de octubre, por el que se establece la ordenación de las enseñanzas universitarias oficiales establece que todos los títulos de Grado finalizarán con la elaboración de un Trabajo Fin de Grado (TFG). En concreto en el apartado 12.7 se determina que "El Trabajo de Fin de Grado tendrá un mínimo de 6 créditos y un máximo del 12,5 por ciento del total de los créditos del título. Deberá realizarse en la fase final del plan de estudios y estar orientado a la evaluación de competencias asociadas al título".

Con ello la ordenación de los grados pretende responder al aprendizaje de competencias como vía de respuesta para atender las necesidades de la sociedad actual, promover la empleabilidad y la inclusión social (Comisión de las Comunidades Europeas, 2001), cuyas competencias clave de referencia se concretaron en la Recomendación 2006/962/CE del Parlamento Europeo y el Consejo, de 18 de diciembre de 2006, sobre las competencias clave para el aprendizaje permanente (Parlamento Europeo y Consejo, 2006).

Cada universidad ha desarrollado un reglamento para establecer la normativa propia que regula y ordena su elaboración.

Por lo general, es una asignatura obligatoria de 6 créditos, a cursar en el último año, pero los objetivos, las competencias y los requisitos para su realización varían de una universidad a otra.

En el caso particular de la Facultad de Ciencias Económicas y Empresariales de la Universidad Loyola Andalucía (ULA) el objetivo del TFG es (ULA, 2014a, p.2):

"Verificar que el estudiante ha adquirido las competencias propias del grado en ADE, tanto generales como específicas y transversales". Al tratarse de una asignatura que debe ser la culminación del grado, se considera que "serán reforzadas las competencias generales. Las competencias específicas dependerán de la temática abordada en cada trabajo fin de grado, y por tanto, del área de conocimiento al que se circunscriben".

La flexibilidad de cada universidad para diseñar esta materia y la novedad de su aplicación nos indujeron a realizar esta investigación, cuyo objetivo es conocer cómo se percibe por parte de los alumnos el enfoque dado a esta asignatura en el grado de ADE de la Universidad Loyola Andalucía, tanto en relación a los aspectos de gestión como a la adquisición de las competencias correspondientes. La información obtenida se utilizará para realizar propuestas de mejora en los procesos educativos y de gestión de esta materia.

Para ello se ha elaborado una encuesta que se envió a los alumnos del grado de ADE del curso 2014-15, que han cursado dicha materia en cualquiera de las convocatorias. La metodología empleada es un estudio descriptivo de los resultados, basado en un análisis de frecuencias y de correlación entre algunas variables. La elección de esta metodología es consecuencia de la limitación del tamaño de la muestra y del objeto de estudio.

\section{LA EVALUACIÓN DE COMPETENCIAS EN LOS TRABAJO FIN DE GRADO}

La evaluación de las competencias constituye una oportunidad de aprendizaje y desarrollo para el alumno (Tejada y Ruiz, 2016). 
Evaluar las competencias (Villa y Poblete, 2007) requiere saber qué se va a evaluar, definir de forma precisa y oportuna cómo se va a evaluar y concretar el nivel de consecución de las competencias que se van a exigir.

El primer requisito se atiende mediante la definición y comunicación de las competencias a través de las guías docentes. Las competencias que se mencionan en la guía docente correspondiente a la asignatura TFG son las que se reflejan en la Tabla 1 (ULA, 2014a, pp.2-3).

Como señalan Snavely y Wright (2003), este tipo de trabajos proporcionan al alumno el contexto en el que, de forma individual, refleja y gana confianza en la integración de todas las competencias que ha ido desarrollando de forma gradual a lo largo de sus estudios, a la vez que le permite profundizar en un tema que le preocupa, acercándole a la realidad.

En cuanto al segundo requisito, relacionado con la definición de forma precisa y oportuna del cómo se va a evaluar, en el caso de nuestra Facultad se ha elaborado una guía orientadora (ULA, 2014c) que se entrega a los tutores y a los alumnos, en la que se establece de forma concreta qué aspectos son claves en la elaboración y desarrollo del TFG, que ponen de manifiesto cómo se interpreta cada una de las competencias en este contexto. En la Tabla 1 se relacionan cada una de las competencias con los aspectos contemplados en la Guía orientativa del TFG que favorecen su consecución. 
TABLA 1. ANÁlISIS de Cómo las COMPETENCIAS SON CONTEMPLADAS EN LA GUía ORIENTATIVA del TFG

\begin{tabular}{|c|c|}
\hline Competencias generales: & $\begin{array}{l}\text { ASPECTOS CONTEMPLADOS EN LA GUIA ORIENTATIVA DEL } \\
\text { TFG }\end{array}$ \\
\hline $\begin{array}{l}\text { - CG1. Toma de decisiones: elegir la } \\
\text { mejor alternativa para actuar } \\
\text { siguiendo un proceso sistemático y } \\
\text { responsabilizándose del alcance y } \\
\text { consecuencias de la opción tomada. }\end{array}$ & $\begin{array}{l}\text { El trabajo fin de grado será siempre un trabajo individual } \\
\text { (p. 3). A lo largo del proceso de realización del trabajo, } \\
\text { el alumno tendrá que tomar distintas decisiones que } \\
\text { condicionarán el alcance y el resultado final de su TFG } \\
\text { (p. 5): } \\
\text { - Elección y análisis del tema objeto de estudio. } \\
\text { - Búsqueda de información revisión de la bibliografía. } \\
\text { - Preparación del primer borrador con el esquema y } \\
\text { cronograma del TFG. } \\
\text { - Desarrollo y redacción del trabajo. } \\
\text { - Elaboración de conclusiones. } \\
\text { lgualmente, la organización del trabajo y la asistencia a } \\
\text { las tutorías académicas programadas a lo largo del } \\
\text { cuatrimestre, para la supervisión del trabajo o la } \\
\text { resolución de dudas metodológicas, formarán parte del } \\
\text { proceso sistemático de realización del TFG y de la toma } \\
\text { de decisiones al respecto (p. 5). }\end{array}$ \\
\hline $\begin{array}{l}\text { - CG2. Orientación al aprendizaje: } \\
\text { utilizar el aprendizaje de manera } \\
\text { estratégica y flexible en función del } \\
\text { objetivo perseguido, a partir del } \\
\text { reconocimiento del propio sistema de } \\
\text { aprendizaje y de la conciencia del } \\
\text { aprendizaje mismo (relacionando la } \\
\text { nueva información con los esquemas } \\
\text { mentales previos y la utilización del } \\
\text { nuevo esquema mental generado). }\end{array}$ & $\begin{array}{l}\text { La propia tipología de los trabajos, reconocida en la } \\
\text { Guía refuerza la necesidad de utilizar el aprendizaje de } \\
\text { manera estratégica y flexible. Los trabajos propuestos } \\
\text { podrán ser, intradisciplinares o interdisciplinares, } \\
\text { atendiendo a los siguientes formatos (p. 3-4): } \\
\text { - Trabajos de revisión e investigación bibliográfica. } \\
\text { - Trabajos empíricos. } \\
\text { - Estudio de casos reales. } \\
\text { - Trabajos de análisis de fuentes de información } \\
\text { secundaria. } \\
\text { - Trabajos de diagnóstico sectorial o empresarial. } \\
\text { Durante el proceso de aprendizaje implícito en la } \\
\text { realización del TFG, el alumno tendrá que desarrollar las } \\
\text { capacidades de juicio, crítica, síntesis o desarrollo, entre } \\
\text { otras. }\end{array}$ \\
\hline $\begin{array}{l}\text { - CG3. Uso de las TIC: utilizar las } \\
\text { Tecnologías de la Información y } \\
\text { Comunicación (TICs) como una } \\
\text { herramienta para la expresión y } \\
\text { comunicación, para el acceso a } \\
\text { fuentes de información, como medio } \\
\text { de archivo de datos y documentos, } \\
\text { para tareas de presentación, para el }\end{array}$ & $\begin{array}{l}\text { La presentación del trabajo durante la defensa requiere } \\
\text { de la utilización de algún medio audiovisual (p. 12). } \\
\text { Según establece la Guía se tendrán en cuenta los } \\
\text { siguientes aspectos relacionados con el uso adecuado } \\
\text { de las tecnologías: } \\
\text { - Claridad de la presentación (coherencia de la } \\
\text { estructura y organización adecuada de la } \\
\text { presentación). }\end{array}$ \\
\hline
\end{tabular}


Mercedes Ruíz Lozano, Pilar Tirado Valencia

La percepción de los alumnos sobre la adquisición de competencias del Trabajo Fin de Grado en ADE. Un análisis en la Universidad Loyola Andalucía

\begin{tabular}{|c|c|}
\hline $\begin{array}{l}\text { aprendizaje, la investigación y el } \\
\text { trabajo cooperativo. }\end{array}$ & $\begin{array}{l}\text { - Visibilidad de la presentación (tamaño de letra, } \\
\text { colores, movimiento...). } \\
\text { - Utilización correcta de cuadros y gráficos } \\
\text { (comprensibles y visibles) } \\
\text { - Coherencia de la presentación con la exposición. } \\
\text { Igualmente, y con el objetivo de facilitar a los } \\
\text { estudiantes el acceso a las bases de datos, fondo } \\
\text { bibliográfico y uso de recursos en la web, así como otros } \\
\text { recursos disponibles a través de las TIC, el servicio de } \\
\text { Biblioteca organiza un curso de formación dirigido a } \\
\text { todos los alumnos del TFG. }\end{array}$ \\
\hline $\begin{array}{l}\text { - CG4. Comunicación verbal: } \\
\text { expresar con claridad y oportunidad } \\
\text { las ideas, conocimientos y } \\
\text { sentimientos propios a través de la } \\
\text { palabra, adaptándose a las } \\
\text { características de la situación y la } \\
\text { audiencia para lograr su comprensión } \\
\text { y adhesión. }\end{array}$ & $\begin{array}{l}\text { En cuanto a la exposición pública del TFG, donde será } \\
\text { necesario poner en juego las competencias } \\
\text { comunicativas, conviene tener en cuenta los siguientes } \\
\text { elementos evaluables (p. 12): } \\
\text { A) EXPOSICIÓN } \\
\text { - Dominio de los conocimientos. } \\
\text { - Distribución del tiempo de la exposición. } \\
\text { - Dominio del lenguaje verbal. } \\
\text { - Dominio del lenguaje disciplinar. } \\
\text { - Capacidad para argumentar. } \\
\text { - Capacidad de síntesis. } \\
\text { - Expresividad. } \\
\text { - Control emocional. } \\
\text { - Claridad en la exposición. } \\
\text { - Actitud ante el público y utilización del medio } \\
\text { - audiovisual. } \\
\text { Resolución de las dudas planteadas por el } \\
\text { Tribual. }\end{array}$ \\
\hline $\begin{array}{l}\text { - CG5. Comunicación escrita: } \\
\text { relacionarse eficazmente con otras } \\
\text { personas a través de la expresión } \\
\text { clara de lo que se piensa y/o siente, } \\
\text { mediante la escritura y los apoyos } \\
\text { gráficos. }\end{array}$ & $\begin{array}{l}\text { A la hora de realizar y evaluar el TFG, el alumno deberá } \\
\text { tener en cuenta los siguientes elementos evaluables (p. } \\
\text { 11): } \\
\text { A) ASPECTOS FORMALES } \\
\text { - Uso adecuado de las normas de estilo recogidas } \\
\text { anteriormente. } \\
\text { - Uso correcto del lenguaje especializado del } \\
\text { campo científico al que pertenezca el TFG. } \\
\text { - Claridad de la redacción. } \\
\text { - Uso óptimo de cuadros y gráficos como soporte } \\
\text { B) Al texto. } \\
\text { - DefeCTOS DE CONTENIDO } \\
\text { - Búsqueda adecuada de indara de objetivos e hipótesis. } \\
\text { documentación sobre el tema seleccionado. y } \\
\text { - Correcta interpretación de la información. } \\
\text { - Descripción óptima de la estructura del trabajo. } \\
\text { - Capacidad de análisis y síntesis. } \\
\text { - Selección, exposición y adecuación de la } \\
\text { metodología a los objetivos planteados. } \\
\text { - Capacidad para aplicar correctamente los } \\
\text { conocimientos y técnicas adquiridas durante la } \\
\text { carrera. }\end{array}$ \\
\hline
\end{tabular}




\begin{tabular}{|c|c|}
\hline & 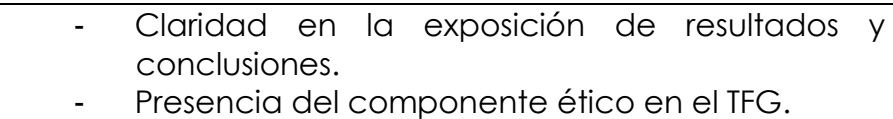 \\
\hline $\begin{array}{l}\text { - CG6. Diversidad e interculturalidad: } \\
\text { comprender y aceptar la diversidad } \\
\text { social y cultural como un componente } \\
\text { enriquecedor personal y colectivo } \\
\text { para desarrollar la convivencia entre } \\
\text { las personas sin incurrir en } \\
\text { discriminación por sexo, edad, religión, } \\
\text { condición social, política y/o étnica. }\end{array}$ & $\begin{array}{l}\text { Entre los temas propuestos por el profesorado, destacan } \\
\text { los relacionados con la diversidad, la igualdad, el } \\
\text { compromiso con los más desfavorecidos y la lucha } \\
\text { contra la discriminación y la pobreza. Estas temáticas son } \\
\text { congruentes con la propia naturaleza de la Universidad } \\
\text { Loyola Andalucía según se establece en sus estatutos. } \\
\text { Algunos aspectos relacionados con la asignación de } \\
\text { temas (art. 3.3. de la Normativa) y de la defensa y } \\
\text { evaluación del TFG (art. } 7 \text { de la Normativa) tratan de } \\
\text { garantizar la transparencia en el proceso y la igualdad } \\
\text { de oportunidades, dentro de la diversidad de trabajos. }\end{array}$ \\
\hline $\begin{array}{l}\text { - CG8. Orientación a la calidad: } \\
\text { buscar la excelencia en la actividad } \\
\text { académica, personal y profesional, } \\
\text { orientada a resultados y centrada en } \\
\text { la mejora continua. }\end{array}$ & $\begin{array}{l}\text { El rigor y los niveles de exigencia en la elaboración y } \\
\text { defensa de los trabajos, el cumplimiento estricto de los } \\
\text { aspectos formales, el cuidado de los detalles, son } \\
\text { expresiones de la búsqueda de la excelencia. } \\
\text { Manifestaciones de esa exigencia podrían ser, según se } \\
\text { establece en la Guía (p. 11): } \\
\text { - Uso adecuado de las normas de estilo recogidas } \\
\text { anteriormente (epígrafe 6.3. de la Guía). } \\
\text { - Uso correcto del lenguaje especializado del campo } \\
\text { científico al que pertenezca el TFG. } \\
\text { - Claridad de la redacción. } \\
\text { - Uso óptimo de cuadros y gráficos como soporte al } \\
\text { texto. }\end{array}$ \\
\hline $\begin{array}{l}\text { - CG10. Innovación: dar una } \\
\text { respuesta satisfactoria a las } \\
\text { necesidades y demandas personales, } \\
\text { organizativas y sociales, modificando } \\
\text { o introduciendo elementos nuevos en } \\
\text { los procesos y en los resultados. }\end{array}$ & $\begin{array}{l}\text { El trabajo fin de grado es siempre un trabajo original e } \\
\text { innovador, sobre un tema propuesto por el tutor. } \\
\text { Conviene remarcar que el tutor, no deja de ser un mero } \\
\text { facilitador y orientador en el proceso de elaboración. } \\
\text { Aunque el resultado final obtenido por el alumno esté } \\
\text { influenciado por este proceso de acompañamiento y } \\
\text { dado que entran en juego muchos elementos no } \\
\text { controlables por el tutor que pueden condicionarlo, la } \\
\text { responsabilidad final del éxito o fracaso del TFG será del } \\
\text { alumno (p. 4). }\end{array}$ \\
\hline Competencias transversales: & $\begin{array}{l}\text { ASPECTOS CONTEMPLADOS EN LA GUIA ORIENTATIVA DEL } \\
\text { TFG }\end{array}$ \\
\hline $\begin{array}{l}\text { - CT1. Sentido ético: sensibilizarse } \\
\text { hacia la dimensión moral inherente a } \\
\text { todo lo humano y lo social (acción } \\
\text { personal, instituciones sociales) e } \\
\text { inclinarse positivamente hacia el bien } \\
\text { moral de uno mismo o de los demás } \\
\text { (vivencia de sentido, realización de la }\end{array}$ & $\begin{array}{l}\text { Además, se valorará especialmente la componente } \\
\text { ética del trabajo. Cualquier proyecto de carácter } \\
\text { universitario, debe estar orientado por una serie de } \\
\text { actitudes y valores éticos compatibles con la } \\
\text { construcción de una sociedad más justa y solidaria. (p. 3) }\end{array}$ \\
\hline
\end{tabular}


Mercedes Ruíz Lozano, Pilar Tirado Valencia

La percepción de los alumnos sobre la adquisición de competencias del Trabajo Fin de Grado en ADE. Un análisis en la Universidad Loyola Andalucía

\begin{tabular}{|l|l|}
\hline persona, sentido de justicia). & \\
\hline Competencias específicas: & $\begin{array}{l}\text { ASPECTOS CONTEMPLADOS EN LA GUIA ORIENTATIVA DEL } \\
\text { TFG }\end{array}$ \\
\hline $\begin{array}{l}\text { CE38. Desarrollar la capacidad para } \\
\text { interrelacionar, en torno a un } \\
\text { determinado objetivo, las enseñanzas } \\
\text { recibidas y las competencias } \\
\text { adquiridas. }\end{array}$ & $\begin{array}{l}\text { La tipología de trabajos fin de Grado definida en la Guía, } \\
\text { requiere que el estudiante desarrolle un espacio de } \\
\text { conocimientos adquiridos durante sus estudios, llevando } \\
\text { a la práctica parte de los conocimientos y habilidades } \\
\text { adquiridos durante su etapa universitaria. Los trabajos } \\
\text { propuestos podrán ser, intradisciplinares } \\
\text { interdisciplinares (p. 3). }\end{array}$ \\
\hline
\end{tabular}

Fuente: Elaborada por los investigadores a partir de la información disponible en la guía docente y en la guía orientativa del TFG

Respecto al requisito de concretar el nivel de consecución de esas competencias que se va a exigir al estudiante, en el caso de nuestra Facultad se han incluido en la guía orientadora (ULA, 2014C) unas hojas de evaluación que se entregan a todos los profesores tutores y posibles miembros del tribunal para que les ayude a realizar su función, aunque no es obligatoria seguirlas al pie de la letra, ni queda cumplimentada formalmente por los evaluadores. En dichos formularios se recogen una serie de ítems evaluables en cada caso, para que el tutor o miembro del tribunal asigne una puntuación concreta y pueda tener así evidencias concretas de la evaluación realizada, de forma similar a lo planteado en otras universidades (Bonilla et al. (2012).

El TFG se realizará bajo la dirección y supervisión del tutor. En relación con el papel del tutor, la guía establece (ULA, 2014c, p.4) que éste deberá:

- Proporcionar apoyo y servir de guía al estudiante durante la realización del trabajo.

- Establecer un cronograma de reuniones en función de las características del trabajo.

- Supervisar el trabajo estableciendo las indicaciones oportunas que permitan al alumno el logro de sus objetivos en tiempo y forma.

- Autorizar el depósito y la defensa del TFG.

Es decir, el tutor tiene la responsabilidad de guiar todo el proceso de aprendizaje del alumno, para garantizar su éxito en línea con los requerimientos planteados por Todd et al. (2006). Pero no asigna una calificación al trabajo, tan sólo tiene la potestad de no autorizar la presentación del trabajo si considera que el nivel no es adecuado. A pesar de ello, el tutor realiza una evaluación auténtica, como denomina Wiggins (1990) al referirse a la preocupación por la integración de las capacidades de los estudiantes, la habilidad por integrar nuevos aprendizajes y dar la misma importancia al proceso como al producto final. Con esta tutorización se responde a una de las mejores prácticas de excelencia identificadas en la realización de TFG (Hernández-Leo et al. 2013) y cuyo objetivo principal es motivar al estudiante, aunque en algunos casos el acompañamiento del tutor en el proceso no es del todo eficaz, como consecuencia de la carga de trabajo del alumno o de su propia organización del trabajo. 
Para contrarrestar esta limitación, como el TFG está previsto en el Plan de Estudios como una actividad de finalización del grado, para su realización se requiere (ULA, 2014b) que al inicio del curso académico al alumno solamente le resten menos de 66 créditos (sin incluir el TFG), si es así, "podrán solicitar a través de los procedimientos establecidos y en el periodo habilitado por el Centro en su calendario académico, la asignación de tema y tutor del TFG", esta asignación sólo tendrá validez durante un año (aunque si el tutor está de acuerdo se mantendrá la asignación durante el siguiente curso académico). La solicitud requiere indicar 10 temas por orden de preferencia entre los ofertados en cada curso académico, teniendo en cuenta que si se ha solicitado por más de un estudiante la asignación se realizará teniendo en cuenta la calificación media de los expedientes académicos de los solicitantes.

El Trabajo Fin de Grado culminará con la redacción de una memoria y su presentación y defensa frente a un tribunal. El tutor deberá informar y autorizar la defensa del TFG. Pero "aun cuando el alumno pueda estar realizando el TFG bajo la supervisión del tutor asignado, no podrá realizar matrícula hasta tanto no haya superado todas las asignaturas obligatorias del Plan de Estudios del Grado" (incluido el nivel mínimo del idioma exigido).

La evaluación del Trabajo Fin de Grado será realizada (ULA, 2014b) por el tribunal designado a tal efecto. La defensa del TFG (ULA, 2014b, p. 4) consiste en "la exposición del alumno sobre los objetivos, metodología y principales conclusiones del Trabajo Fin de Grado, que no podrá superar los 15 minutos, así la duración total del acto público de exposición y defensa no podrá superar los 30 minutos". Esta evaluación está en proceso de revisión puesto que diversos estudios (Hernández-Leo et al., 2013; Mateo et al. 2012; Valderrama et al. 2010) han puesto de manifiesto la relevancia de que se haga una evaluación conjunta, en la que participe tanto el tutor como el tribunal evaluador, e incluso otros actores, para que la evaluación responda claramente al desarrollo de competencias alcanzado.

Con la elaboración y difusión de documentos como la guía docente, la guía orientadora, las fichas de evaluación, se pretende ser transparentes y garantizar homogeneidad y objetividad en la materia. Pero las características del TFG hacen que haya una gran diversidad de objetivos que atender, de temas a desarrollar, de tutores, de enfoques, etc., que finalmente tienen que ser evaluados por diversos tribunales. Todo ello puede provocar diferencias sustantivas entre unos trabajos y otros en cuanto a su desarrollo y evaluación.

De manera informal, dependiendo de la relación alumno-tutor, en consonancia con los resultados obtenidos por Gallardo y Reyes (2010), el alumno recibe por parte de su tutor un informe periódico de cómo va evolucionando en la adquisición de las diferentes competencias a desarrollar con la elaboración de sU TFG, proceso que favorece unos resultados excelentes, como pusieron de manifiesto Alfaro Tanco et al. (2014).

Así mismo, en el momento de la defensa del TFG, los distintos miembros del tribunal asumen un papel en la evaluación y realizan, de forma verbal, una evaluación pormenorizada de las distintas competencias, tal y como queda reflejado en la Tabla 2:

Tabla 2. Evaluación en la defensa del Trabajo Fin de Grado

\begin{tabular}{|l|l|}
\hline Miembro del Tribunal & \multicolumn{1}{c|}{ Competencias } \\
\hline Presidente & CG1, CG6, CG8, CG10, CT1, CE38 \\
\hline Vocal & CG2, CG3, CG4, CG5, CG8 \\
\hline
\end{tabular}


Mercedes Ruíz Lozano, Pilar Tirado Valencia

La percepción de los alumnos sobre la adquisición de competencias del Trabajo Fin de Grado en ADE. Un análisis en la Universidad Loyola Andalucía

\begin{tabular}{|l|l|}
\hline Secretario & CG2, CG3, CG5, CG6, CG8, CT1 \\
\hline
\end{tabular}

Fuente: Elaboración propia

Ante esta situación y dada la novedad del enfoque en la metodología y en la evaluación de esta asignatura, tanto para el estudiante como para el profesor tutor, nos preocupaba conocer cómo es percibida esta diversidad por parte del alumnado en el curso 2014-2015. Hay que tener en cuenta que el alumno tendrá su percepción y expectativas, así como una valoración muy subjetiva de sus capacidades y motivaciones (Coll et al. 2008, Robledo et al. 2015), pero se considera que un análisis global y continuado en el tiempo puede ayudar a establecer un proceso de mejora en el transcurso de la realización y de la gestión de los TFG.

El conocer si los procesos de gestión relacionados con la implementación de esta materia está influyendo en la consecución de las competencias identificadas, así como si los documentos (guía docente y formularios establecidos para la evaluación) que se utilizan para comunicar y evaluar dichas competencias están favoreciendo su consecución, o si por el contrario está siendo influido por la falta de atención de uno de los requisitos establecido por Snavely y Wright (2003) sobre la necesidad de que se desarrolle un tema que preocupe al alumno, o por otro tipo de circunstancias académicas, pusieron de manifiesto la necesidad de responder las siguientes cuestiones:

- ¿ ¿cuáles son las temáticas preferentes para el desarrollo del TFG por los alumnos de ADE?

- $\quad \dot{s}$ se atienden los deseos del alumno en cuanto a la temática que le gustaría desarrollar?

- $\quad$ influye el número de créditos matriculados en ese curso en el momento de la defensa del TFG y en la nota obtenida?

- ¿̇cuál es la percepción del alumno en cuanto a la aportación que ha supuesto el TFG en el desarrollo de sus competencias?

- $\quad$ én qué ha consistido el desarrollo de esas competencias? En cuanto a la búsqueda de la información, a la expresión escrita, a la orientación a la calidad, al nivel de innovación, al proceso de reflexión que le ha supuesto la elaboración del TFG,...

- ¿̇cuál ha sido el nivel de satisfacción con todo el proceso de desarrollo y defensa del TFG?

\section{METODOLOGÍA}

Para responder a estas cuestiones se ha elaborado una encuesta que se ha hecho llegar a los alumnos que han defendido su TFG para finalizar el curso 2014-2015, tanto en la convocatoria ordinaria como en la convocatoria extraordinaria.

El ámbito de estudio ha estado formado por los 100 TFG matriculados en esta materia durante el curso 2014-15, con los que se ha contactado a través de su correo electrónico de la universidad una vez que han sido informados de la nota obtenida, enviándoseles un segundo mensaje recordatorio para animarles a responder. Finalmente, la muestra se compone de 37 alumnos que han respondido a la encuesta, lo que representa un índice de respuesta del $37 \%$. 
El análisis de la percepción de los estudiantes se ha utilizado ya en estudios previos en este ámbito (Rubio et al. 2016; Torres-Coronas y Vidal-Blasco, 2015; Rothwell y Arnold, 2007).

La investigación consiste en un estudio empírico, a pequeña escala y exploratorio, para conocer la experiencia de los alumnos que han vivido, durante este curso académico 2014-2015, el reto de elaborar y defender un TFG (es el segundo año que se realiza en la Facultad de Administración y Dirección de Empresas de la Universidad Loyola Andalucía con la implantación de los nuevos grados).

Para su realización se desarrolló una encuesta específicamente diseñada para responder a las cuestiones que son objeto de este estudio. En esta se pedía a los alumnos que realizaran una autoevaluación del nivel de adquisición de las competencias con cuestiones que respondían a una escala de Likert de cinco categorías, e información sobre el contexto en el que los alumnos habían cursado esta materia.

Este instrumento de autoevaluación ha sido valorado como muy adecuado para implicar al estudiante en su proceso de formación, ya que fomenta la crítica y la reflexión (Rodríguez et al. 2008), en línea con las competencias planteadas por esta materia.

Un estudio descriptivo de los resultados permitirá dar respuesta a los interrogantes planteados en esta investigación. La metodología empleada ha sido un estudio de frecuencias y de correlación entre algunas variables.

\section{RESULTADOS}

Los alumnos que han atendido la encuesta presentan las siguientes características (Tabla 3): 
Tabla 3 Característica de la muestra

\begin{tabular}{|c|l|c|c|}
\hline \multirow{2}{*}{ Sexo } & Varón & No alumnos & $\%$ \\
\cline { 2 - 4 } & Mujer & 21 & $57 \%$ \\
\hline \multirow{4}{*}{\begin{tabular}{c} 
Edad \\
\cline { 2 - 4 }
\end{tabular}} & $21-22$ & 16 & $43 \%$ \\
\cline { 2 - 4 } & $23-25$ & 12 & $32 \%$ \\
\cline { 2 - 4 } & $>25$ & 15 & $41 \%$ \\
\hline \multirow{2}{*}{$\begin{array}{c}\text { Créditos } \\
\text { pendientes }\end{array}$} & 448 & 15 & $27 \%$ \\
\cline { 2 - 4 } & $48-60$ & 19 & $51 \%$ \\
\cline { 2 - 4 } & $>60$ & 3 & $8 \%$ \\
\hline \multirow{2}{*}{\begin{tabular}{c} 
Convocatoria \\
\cline { 2 - 4 }
\end{tabular}} & Ordinaria & 14 & $38 \%$ \\
\cline { 2 - 4 } & Extraordinaria & 23 & $62 \%$ \\
\hline
\end{tabular}

Fuente: Elaboración propia

De las características de la muestra resalta que predomina el alumno que termina más o menos en la edad propia de finalización de sus estudios de grado, son pocos los alumnos que han retrasado el momento de finalización porque entre los 10 alumnos que presentan una edad superior a 25 años se pueden encontrar algunos provenientes de la antigua Diplomatura en Ciencias Empresariales que al cabo de los años han realizado el curso de adaptación al grado.

Asimismo, destaca que los alumnos que en su último curso tienen una carga de créditos superior a un curso normal (60 créditos) difícilmente son capaces de realizar y defender el TFG en el mismo curso académico. Lo que además se pone de manifiesto en que sólo el 38 \% de los encuestados lo han presentado en la convocatoria ordinaria. Todo ello muestra la dificultad que supone al alumno la realización y defensa del TFG.

En cuanto a las temáticas preferentes que han sido desarrolladas y defendidas por los alumnos durante el curso 2014-2015, en la Tabla 4 se puede observar que ha habido una gran variedad, siendo las menos representadas las áreas de Marketing y de Ética y Humanismo. 
Mercedes Ruíz Lozano, Pilar Tirado Valencia La percepción de los alumnos sobre la adquisición de competencias del Trabajo Fin de Grado en ADE. Un análisis en la Universidad Loyola Andalucía

Tabla 4. Distribución TFG por Áreas de Conocimiento

\begin{tabular}{|l|c|c|}
\hline \multicolumn{1}{|c|}{ Áreas de Conocimiento } & $\begin{array}{c}N^{\circ} \\
\text { alumnos }\end{array}$ & $\%$ \\
\hline Derecho & 7 & $18,92 \%$ \\
\hline Gestión Empresarial & 7 & $18,92 \%$ \\
\hline Finanzas y Contabilidad & 7 & $18,92 \%$ \\
\hline Marketing & 3 & $8,11 \%$ \\
\hline Economía & 9 & $24,32 \%$ \\
\hline Ética y Humanismo & 4 & $10,81 \%$ \\
\hline Total & 37 & $100 \%$ \\
\hline
\end{tabular}

Fuente: Elaboración propia

Sin embargo, esta distribución se podría ver afectada por la oferta de temas que se realiza por parte del claustro, por ello se ha preguntado cuál ha sido la opción en la que el alumno había seleccionado el tema desarrollado (el alumno debe elegir hasta 10 temas en su solicitud por orden de prioridad). Como se puede observar en la Tabla 5 , el $51,35 \%$ de los alumnos han desarrollado un tema que han elegido en primera 0 segunda opción, sólo un 27,04\% de los alumnos han debido hacerlo en temas que habían elegido en una opción superior a la quinta.

Tabla 5. Análisis de la opción en la que los alumnos han elegido el tema desarrollado en su TFG

\begin{tabular}{|l|c|c|}
\hline $\begin{array}{c}\text { Orden de la opción en la } \\
\text { elección del tema (1-10) }\end{array}$ & $\begin{array}{c}\mathrm{N}^{\circ} \\
\text { alumnos }\end{array}$ & $\%$ \\
\hline Primera & 16 & $43,24 \%$ \\
\hline Segunda & 3 & $8,11 \%$ \\
\hline Tercera & 2 & $5,41 \%$ \\
\hline Cuarta & 3 & $8,11 \%$ \\
\hline Quinta & 3 & $8,11 \%$ \\
\hline Séptima & 2 & $5,41 \%$ \\
\hline Novena & 2 & $5,41 \%$ \\
\hline Décima & 6 & $16,22 \%$ \\
\hline Total & 37 & $100 \%$ \\
\hline
\end{tabular}

Fuente: Elaboración propia

Todos los trabajos presentados son aprobados porque se ha diseñado un mecanismo que realiza un doble filtro antes de la defensa: para el depósito se requiere de la autorización por parte del profesor tutor, que a su vez se ve reforzado por el control del presidente del tribunal evaluador antes de la realización de la defensa, ya que si éste viera que el TFG no presenta un nivel adecuado se habla con el tutor y se solicita al alumno que lo retire y lo revise antes de proceder a su defensa. Las calificaciones obtenidas en el curso 2014-2015 se resumen en la Tabla 6, en la que se puede observar que predomina la nota media de notable.

Tabla 6. Calificaciones medias obtenidas en los TFG en el curso 2014-2015. 
Mercedes Ruíz Lozano, Pilar Tirado Valencia

La percepción de los alumnos sobre la adquisición de competencias del Trabajo Fin de Grado en ADE. Un análisis en la Universidad Loyola Andalucía

\begin{tabular}{|l|c|c|c|c|}
\hline Convocatoria/Calificación & Aprobado & Notable & Sobresaliente & Total \\
\hline Ordinaria & 2 & 6 & 6 & 14 \\
\hline Extraordinaria & 4 & 13 & 6 & 23 \\
\hline Total & 6 & 19 & 12 & 37 \\
\hline
\end{tabular}

Fuente: Elaboración propia

En cuanto al nivel de influencia de la carga de créditos en la calificación obtenida se ha realizado un análisis de correlaciones y no se ha encontrado incidencia estadística entre ambas variables. Sin embargo, si se ha encontrado correlación estadísticamente significativa $(0,654)$ entre el orden de la opción en la elección del tema asignado y la calificación obtenida, de manera que la calificación ha sido inferior a medida que el orden de la opción del tema ha sido superior.

En relación a la percepción que tiene el alumno en cuanto a la influencia de la realización del TFG en la mejora de sus competencias (Tabla 7, en la que los ítems analizados se relacionan con las competencias identificadas en la Tabla 1 para su mejor comprensión), se puede observar que el alumno realiza una valoración alta (en torno a 4 sobre 5) de cómo el TFG ha supuesto una mejora de sus competencias, salvo en la competencia de Innovación, quizás porque no se ha interpretado bien ese término en el contexto de la realización del TFG. Por otro lado, no ha sido muy alta la valoración en cuanto al uso de las TICs, quizás porque el alumno actual es un usuario constante de las nuevas tecnologías por lo que no le ha supuesto ningún cambio su uso en la elaboración del TFG. Así mismo, nos sorprende la valoración de la incidencia en la competencia de comunicación oral porque todos han tenido que realizar una defensa oral, sólo se puede explicar esta valoración por el hecho de que son muchas las materias en nuestro grado en las que tienen que hacer una presentación de un trabajo o de un tema, por lo que ellos perciben que ésta es una competencia que han desarrollado ya anteriormente en otras materias. 
Mercedes Ruíz Lozano, Pilar Tirado Valencia

La percepción de los alumnos sobre la adquisición de competencias del Trabajo Fin de Grado en ADE. Un análisis en la Universidad Loyola Andalucía

Tabla 7. Valoración media de los alumnos en cuanto a mejora de sus competencias

\begin{tabular}{|l|c|}
\hline \multicolumn{1}{|c|}{ Competencia } & $\begin{array}{c}\text { Valor medio } \\
(1-5)\end{array}$ \\
\hline Búsqueda de información (CG1, CG5) & 4,00 \\
\hline Organización e interpretación de datos (CG3) & 3,78 \\
\hline Comunicación escrita (CG5) & 3,97 \\
\hline Comunicación oral (CG4) & 3,59 \\
\hline Utilización de las TICS (CG3) & 3,51 \\
\hline Orientación a la calidad (CG8) & 4,03 \\
\hline Proceso de reflexión de un tema (CE38) & 4,00 \\
\hline $\begin{array}{l}\text { Juicio lógico, crítico y creativo de un tema } \\
\text { (CG2) }\end{array}$ & 3,97 \\
\hline Innovación (CG10) & 3,22 \\
\hline Compromiso profesional (CG8) & 3,92 \\
\hline
\end{tabular}

Fuente: Elaboración propia

Por otra parte, los resultados del estudio demuestran que la percepción que tiene el estudiante sobre su nivel de asimilación de competencias está correlacionado con la calificación obtenida, aunque esta correlación aun siendo estadísticamente significativa, no sea muy alta $(0,421)$.

De forma específica se ha tratado de investigar cómo se han desarrollado estas competencias, lo que nos permitirá conocer cuáles son las fortalezas o debilidades percibidas por los alumnos.

En relación a la "Búsqueda de información" (Tabla 8), se puede resaltar que, en general, se han consultado diferentes fuentes de información y que ello les ha supuesto un gran esfuerzo y ha requerido bastante tiempo al alumno, respondiendo en gran medida a los objetivos del TFG. Aunque ha sido ayudado por parte del tutor como se refleja en esa valoración media en el epígrafe "la bibliografía le ha sido proporcionada" y que se ha tendido más a la consulta de artículos académicos frente a la utilización de artículos cortos y de noticias, por lo que se observa una cierta orientación de los TFG a estar fundamentados en un enfoque básico de las materias, muy académico. 
Mercedes Ruíz Lozano, Pilar Tirado Valencia

La percepción de los alumnos sobre la adquisición de competencias del Trabajo Fin de Grado en ADE. Un análisis en la Universidad Loyola Andalucía

Tabla 8. Desarrollo de la competencia Búsqueda de Información

\begin{tabular}{|l|c|}
\hline \multicolumn{1}{|c|}{ Aspectos } & $\begin{array}{c}\text { Valor medio } \\
\text { (1-5) }\end{array}$ \\
\hline Ha realizado una revisión de diferentes fuentes de información & 4,08 \\
\hline La bibliografía utilizada le ha sido proporcionada & 2,89 \\
\hline $\begin{array}{l}\text { La bibliografía consultada han sido fundamentalmente artículos } \\
\text { cortos y noticias sacadas de internet y no artículos académicos }\end{array}$ & 2,22 \\
\hline $\begin{array}{l}\text { La búsqueda de bibliografía ha supuesto una dedicación de } \\
\text { tiempo muy importante en la elaboración del trabajo }\end{array}$ & 3,95 \\
\hline
\end{tabular}

Fuente: Elaboración propia

En relación a la "Organización e interpretación de datos", es de resaltar que no todos los TFG responden al desarrollo de esta competencia, por lo que se ha pedido a los estudiantes que identificaran el tipo de estudio realizado con el TFG para conocer en cuántos casos se ha realizado un análisis de datos y de qué tipo. Como se puede observar en la Tabla 9, sólo 8 alumnos se han limitado a realizar un estudio de revisión bibliográfica, el resto ha hecho un análisis de datos, ya sea descriptivo y/o cuantitativo. Por lo que se puede decir que en el $78 \%$ de los casos analizados se ha desarrollado esta competencia.

Tabla 9. Tipo de estudio realizado en el TFG

\begin{tabular}{|l|c|c|}
\hline \multicolumn{1}{|c|}{ Aspectos } & No Alumnos & $\%$ \\
\hline $\begin{array}{l}\text { El TFG responde únicamente a un modelo de revisión } \\
\text { bibliográfica }\end{array}$ & 8 & $22 \%$ \\
\hline $\begin{array}{l}\text { Ha realizado un análisis de datos fundamentalmente } \\
\text { descriptivo }\end{array}$ & 10 & $27 \%$ \\
\hline $\begin{array}{l}\text { Ha realizado un análisis de datos fundamentalmente } \\
\text { cuantitativo }\end{array}$ & 13 & $35 \%$ \\
\hline Ha realizado un análisis descriptivo y cuantitativo & 16 & $43 \%$ \\
\hline
\end{tabular}

Fuente: Elaboración propia

En relación a la "Comunicación escrita", nos preocupaba conocer el esfuerzo que ha supuesto para el alumno realizar de forma individual un trabajo de esta extensión. Sin embargo, como se puede observar en la Tabla 10, los alumnos han valorado por debajo de la media este esfuerzo (se han sentido poco identificados con estos aspectos), no considerando que la elaboración de ese trabajo haya sido digna de resaltar. No obstante, los resultados relacionados con esta competencia pueden ser una muestra de la falta de exigencia y rigor por parte del alumnado en la comunicación escrita, o como consecuencia de que a lo largo del grado se ha trabajado lo suficiente esta competencia como para que ellos perciban que en este momento no les ha requerido un mayor esfuerzo. 
Mercedes Ruíz Lozano, Pilar Tirado Valencia

La percepción de los alumnos sobre la adquisición de competencias del Trabajo Fin de Grado en ADE. Un análisis en la Universidad Loyola Andalucía

Tabla 10. Valoración del desarrollo de la competencia Comunicación Escrita

\begin{tabular}{|l|c|}
\hline \multicolumn{1}{|c|}{ Aspectos } & $\begin{array}{c}\text { Valor medio } \\
\text { (1-5) }\end{array}$ \\
\hline $\begin{array}{l}\text { Ha requerido el apoyo del tutor o persona ajena para poder } \\
\text { redactar correctamente el trabajo }\end{array}$ & 2,68 \\
\hline $\begin{array}{l}\text { Ha supuesto un esfuerzo importante por la necesidad de resumir y } \\
\text { expresarlo con otras palabras }\end{array}$ & 3,46 \\
\hline Ha supuesto un esfuerzo importante el expresar las ideas básicas & 2,89 \\
\hline No ha supuesto un esfuerzo superior al realizado en otras materias & 2,24 \\
\hline
\end{tabular}

Fuente: Elaboración propia

En cuanto al desarrollo de la competencia "Comunicación oral", se ha querido conocer cómo se ha sentido en el momento de la defensa. Así en la Tabla 11, se puede comprobar que se ha dado una valoración baja a estos aspectos, mostrándose poco identificados con una situación de nervios o estrés, de acuerdo con la percepción reflejada en relación a esta competencia en el análisis global. Aunque por otro lado, esta percepción no se ha visto corroborada con la valoración que muestra el tercer ítem, acerca del proceso de formación en relación a esta competencia, lo que se podría interpretar, por un lado, como respuesta a la práctica adquirida en otras materias, y por otro, consecuencia del hecho de ir bien preparado.

Tabla 11. Valoración de la situación ante la defensa del TFG

\begin{tabular}{|l|c|}
\hline \multicolumn{1}{|c|}{ Aspectos } & $\begin{array}{c}\text { Valor medio } \\
(1-5)\end{array}$ \\
\hline Ha sido influida por los nervios & 2,35 \\
\hline \begin{tabular}{l} 
La limitación del tiempo le ha generado mayor estrés \\
\hline $\begin{array}{l}\text { Se ha sentido cómodo porque ha sido un paso más en el proceso } \\
\text { de formación llevado a cabo en otras materias del Grado }\end{array}$
\end{tabular} & 2,46 \\
\hline
\end{tabular}

Fuente: Elaboración propia

En relación a la "Utilización de las TICs", como ya se ha mencionado anteriormente, los alumnos actuales son usuarios asiduos de las nuevas tecnologías, cuestión que se ha ratificado al observarse que una gran mayoría ha utilizado software diverso en el desarrollo y defensa del TFG (Tabla 12). 
Mercedes Ruíz Lozano, Pilar Tirado Valencia

La percepción de los alumnos sobre la adquisición de competencias del Trabajo Fin de Grado en ADE. Un análisis en la Universidad Loyola Andalucía

Tabla 12. Análisis de la utilización de las TICs

\begin{tabular}{|l|c|c|}
\hline \multicolumn{1}{|c|}{ Aspectos } & No Alumnos & $\%$ \\
\hline Ha utilizado exclusivamente un procesador de textos & 3 & $8 \%$ \\
\hline $\begin{array}{l}\text { Ha utilizado procesador de textos, programas de hoja de } \\
\text { cálculo }\end{array}$ & 2 & $5 \%$ \\
\hline Ha utilizado procesador de textos, programa de presentación & 11 & $30 \%$ \\
\hline $\begin{array}{l}\text { Ha utilizado programas de procesador de textos, hojas de } \\
\text { cálculo, y de presentación }\end{array}$ & 18 & $49 \%$ \\
\hline $\begin{array}{l}\text { Ha utilizado programas de procesador de textos, hojas de } \\
\text { cálculo, de presentación y software estadístico }\end{array}$ & 3 & $8 \%$ \\
\hline
\end{tabular}

Fuente: Elaboración propia

En relación a la "Orientación a la calidad", nos preocupaba conocer si el alumno se ha sentido presionado por la carga de trabajo $u$ otras circunstancias para desarrollar el trabajo en tiempo y forma con una orientación de calidad. En la Tabla 13 se puede observar que la valoración ha sido más bien alta en cuanto a que el alumno cree que ha podido desarrollar el TFG atendiendo a los plazos marcados. Además, no parece que otro tipo de actividades académicas hayan interferido en la realización del trabajo. Estos resultados son congruentes con la carga de trabajo (créditos matriculados en este curso) ya que como se ha comentado anteriormente la mayoría presenta una carga inferior a los 60 créditos previstos en un curso normal.

Tabla 13. Análisis de los aspectos que han podido influir en la orientación a la calidad.

\begin{tabular}{|l|c|}
\hline \multicolumn{1}{|c|}{ Aspectos } & $\begin{array}{c}\text { Valor medio } \\
(1-5)\end{array}$ \\
\hline $\begin{array}{l}\text { Ha podido desarrollar el TFG atendiendo los plazos marcados } \\
\text { por el tutor }\end{array}$ & 3,73 \\
\hline $\begin{array}{l}\text { La presión de otros exámenes o actividades han impedido } \\
\text { desarrollar el TFG con los niveles de calidad deseados }\end{array}$ & 2,68 \\
\hline $\begin{array}{l}\text { La realización de unas prácticas han impedido desarrollar el } \\
\text { TFG con los niveles de calidad deseados }\end{array}$ & 2,43 \\
\hline $\begin{array}{l}\text { La incertidumbre acerca de la finalización de los estudios y el } \\
\text { cumplimiento de los requisitos para la matriculación del } \\
\text { trabajo, ha condicionado el ritmo de desarrollo }\end{array}$ & 2,30 \\
\hline
\end{tabular}

Fuente: Elaboración propia

En relación al "Proceso de reflexión acerca de un tema", como se puede observar en la Tabla 14, por regla general, el alumno ha reflexionado poco en relación con el tema asignado, en algunos casos se han sentido identificados con la opción de no limitarse a obtener un conocimiento teórico del tema y han tratado de realizar una aplicación práctica. 
Mercedes Ruíz Lozano, Pilar Tirado Valencia

La percepción de los alumnos sobre la adquisición de competencias del Trabajo Fin de Grado en ADE. Un análisis en la Universidad Loyola Andalucía

Tabla 14. Análisis del proceso de reflexión acerca del tema desarrollado en el TFG

\begin{tabular}{|l|c|}
\hline \multicolumn{1}{|c|}{ Aspectos } & $\begin{array}{c}\text { Valor medio } \\
(1-5)\end{array}$ \\
\hline $\begin{array}{l}\text { El TFG ha sido un resumen de bibliografía diversa con la que no } \\
\text { he sido interpelado }\end{array}$ & 2,24 \\
\hline $\begin{array}{l}\text { La revisión de la bibliografía ha requerido una redefinición del } \\
\text { enfoque del tema y una propuesta original en su TFG }\end{array}$ & 2,92 \\
\hline La revisión bibliográfica ha permitido una aplicación práctica & 3 \\
\hline
\end{tabular}

Fuente: Elaboración propia

En relación a la "Realización de un juicio lógico, crítico y creativo acerca de un tema", se ha preguntado a los alumnos que valoren cómo se han sentido de identificados con la aplicación de esta competencia. Los resultados ponen de manifiesto (Tabla 15) que el TFG ha supuesto un desarrollo medio de esta competencia, resaltando que el valor más alto se presenta en las implicaciones sociales y éticas, fruto quizás de la orientación dada por los tutores a los temas ofertados.

Tabla 15. Análisis del desarrollo de la competencia juicio lógico, crítico y creativo

\begin{tabular}{|l|c|}
\hline \multicolumn{1}{|c|}{ Aspectos } & $\begin{array}{c}\text { Valor medio } \\
(1-5)\end{array}$ \\
\hline $\begin{array}{l}\text { El análisis realizado ha permitido reforzar o criticar posturas de } \\
\text { algunos autores o analizar diferentes propuestas }\end{array}$ & 3 \\
\hline $\begin{array}{l}\text { El estudio ha permitido poner de manifiesto implicaciones } \\
\text { sociales y/o éticas }\end{array}$ & 3,05 \\
\hline El estudio ha permitido proponer soluciones argumentadas & 2,78 \\
\hline
\end{tabular}

Fuente: Elaboración propia

El nivel de "Innovación del TFG" se ha interpretado en el sentido de que haya supuesto un avance en su conocimiento de alguna materia o que le haya permitido acercase a temas prioritarios para la sociedad. En este sentido, el alumno ha hecho una valoración (Tabla 16) que tiende a ser algo mayor cuando el tema ha resultado de su interés. La innovación y el interés por el tema elegido ha sido una de las competencias que en el estudio ha resultado tener una correlación significativa con la calificación obtenida $(0,434)$. 
Mercedes Ruíz Lozano, Pilar Tirado Valencia

La percepción de los alumnos sobre la adquisición de competencias del Trabajo Fin de Grado en ADE. Un análisis en la Universidad Loyola Andalucía

Tabla 16. Análisis de la percepción por parte del alumno del nivel de innovación del TFG

\begin{tabular}{|l|c|}
\hline \multicolumn{1}{|c|}{ Aspectos } & $\begin{array}{c}\text { Valor medio } \\
(1-5)\end{array}$ \\
\hline $\begin{array}{l}\text { El desarrollo del TFG ha supuesto una novedad en su proceso } \\
\text { de formación }\end{array}$ & 3,16 \\
\hline $\begin{array}{l}\text { El desarrollo del TFG le ha permitido profundizar en temas de su } \\
\text { interés }\end{array}$ & 3,78 \\
\hline $\begin{array}{l}\text { El desarrollo del TFG ha supuesto atender temas y } \\
\text { problemáticas novedosas de interés para los demás o para la } \\
\text { sociedad en general }\end{array}$ & 3,41 \\
\hline
\end{tabular}

Fuente: Elaboración propia

Con respecto a la influencia en "Competencia profesional", nos ha parecido oportuno que más que limitarnos al análisis de una competencia específica de conocimiento, lo que se pretenda sea fomentar el compromiso y la responsabilidad con una tarea que tiene que realizar por sí mismo aunque cuente con la ayuda o guía del tutor. Por ello, nos planteamos conocer la valoración que el alumno hace de sí mismo en cuanto al compromiso que ha tenido en relación con el desarrollo del TFG. Resalta (Tabla 17) que el $76 \%$ de los alumnos han reconocido haber sido tutorizados en la realización de sU TFG y que el $62 \%$ de los participantes en el estudio reconocen haber sido influidos en su compromiso con el rigor y la búsqueda de la calidad.

Tabla 17. Análisis de la influencia en la "competencia profesional"

\begin{tabular}{|l|c|c|}
\hline \multicolumn{1}{|c|}{ Aspectos } & No Alumnos & $\%$ \\
\hline $\begin{array}{l}\text { Ha asistido regularmente a las tutorías de manera periódica y ha } \\
\text { ido siguiendo las propuestas concretas de su tutor }\end{array}$ & 28 & $76 \%$ \\
\hline Ha trabajado regularmente en su elaboración & 17 & $46 \%$ \\
\hline Ha trabajado de vez en cuando en su elaboración & 4 & $11 \%$ \\
\hline Ha realizado el grueso del trabajo en los últimos días & 3 & $8 \%$ \\
\hline $\begin{array}{l}\text { La realización del trabajo ha aumentado su compromiso por el } \\
\text { rigor y la búsqueda de la calidad, por ejemplo, al tener que } \\
\text { respetar unas normas de estilo }\end{array}$ & 23 & $62 \%$ \\
\hline
\end{tabular}

Fuente: Elaboración propia

Sin embargo, es de resaltar que, a pesar de la insistencia de que el TFG debe ser un trabajo desarrollado gradualmente a lo largo del curso para tratar de favorecer la capacidad de maduración y profundización en el tema elegido, sólo el $46 \%$ de los participantes del estudio reconocen haber trabajado regularmente en su desarrollo.

Por último, se ha querido conocer cuál es el "Grado de satisfacción del alumno en relación a todo el proceso relacionado con el TFG". En la Tabla 18 se puede observar que el nivel de satisfacción ha sido medio-alto, resaltando cómo el alumno valora muy alto el trabajo desarrollado y que no ve totalmente reflejado su esfuerzo en la 
calificación final, por ello este último es el epígrafe con la valoración más baja, 3,30 (en todo caso por encima de la media).

Tabla 18. Análisis de la satisfacción global en relación con distintos aspectos del TFG

\begin{tabular}{|l|c|}
\hline \multicolumn{1}{|c|}{ Aspectos } & $\begin{array}{c}\text { Valor medio } \\
(1-5)\end{array}$ \\
\hline El proceso de acompañamiento del tutor & 4 \\
\hline El trabajo autónomo desarrollado por mí mismo & 4,19 \\
\hline El desarrollo de la defensa del trabajo & 3,84 \\
\hline El nivel de competencias adquirido & 3,57 \\
\hline La calificación obtenida & 3,30 \\
\hline
\end{tabular}

Fuente: Elaboración propia

Esta valoración se ve reforzada por la correlación estadísticamente significativa que se ha observado entre la valoración media de satisfacción global de cada alumno con la calificación obtenida $(0,678)$.

\section{CONCLUSIONES}

El desarrollo y la evaluación de los TFG en los planes de estudio están suponiendo todo un reto para el claustro de las universidades. La novedad de la asignatura y su enfoque la dotan de una gran cantidad de particularidades que son fuente de oportunidades en el proceso de aprendizaje, pero también de riesgos debido a su enorme diversidad y especificidades.

La experiencia en los últimos años ha puesto de manifiesto la complejidad del proceso de elaboración del trabajo, la distinta casuística en cuanto a temas, competencias a evaluar y diversidad de profesorado para una misma materia, más aún cuando no todo el profesorado posee la misma formación a este respecto (Ion y Cano, 2012). La evaluación también se ha convertido en un asunto salpicado de múltiples matices. Todo ello nos ha llevado a preocuparnos por conocer cuál es la percepción del alumno en relación a su proceso de aprendizaje y adquisición de competencias en esta materia.

Según nuestros resultados, la realización del TFG está suponiendo un retraso en la finalización de los estudios de grado, ya que solo el 38 \% de los alumnos que han participado en el estudio en el curso 2014-2015 se han presentado en la convocatoria ordinaria, en línea con los resultados encontrados por Bonilla et al. (2012). El porcentaje es muy inferior si se consideran la totalidad de trabajos defendidos este curso (19\%), y menor aún si se toman en cuenta la totalidad de los trabajos asignados.

En torno al $50 \%$ de los alumnos han desarrollado un tema que han elegido en primera o segunda opción, por lo que se considera que todavía es necesario avanzar más en la oferta de temas para intentar acercar las expectativas del alumno a la oferta del profesorado. Sobre todo después de observar que el interés del alumno y el carácter innovador o novedoso del tema está relacionado con la calificación obtenida. En una futura investigación se podría analizar cuáles son las áreas y temas preferentes de los alumnos y cuáles son los factores que pueden estar influyendo en esa elección. 
Mercedes Ruíz Lozano, Pilar Tirado Valencia

La percepción de los alumnos sobre la adquisición de competencias del Trabajo Fin de Grado en ADE. Un análisis en la Universidad Loyola Andalucía

La percepción por parte del alumno sobre el grado de adquisición de las competencias, en general, es positiva, así como del nivel de satisfacción con el proceso. Sin embargo, llaman la atención los escasos resultados en cuanto a la orientación hacia la calidad, el juicio crítico y el grado de compromiso. Este último podría ser fruto de la precipitación de muchos trabajos que se presentan sin haber alcanzado el nivel de madurez suficiente por la premura y el deseo de finalizar los estudios dentro del mismo curso académico.

Muchas de estas deficiencias en la percepción de los estudiantes encuestados son ya imposibles de solventar, como consecuencia de que, como se ha descrito, en este momento el alumno sólo recibe formalmente y por escrito una nota como evaluación del trabajo realizado, por lo que se llama la atención sobre la necesidad de establecer mecanismos formales para el feedback y evaluación de las distintas competencias, de acuerdo con lo argumentado por Tejada y Ruiz (2016).

Así mismo, se ha observado una diferencia entre la satisfacción del alumno con su trabajo y la satisfacción con la calificación obtenida (en línea con lo planteado por Coll et al. 2008; Rubio et al. 2016), fruto quizás de un desconocimiento de los parámetros evaluados y del nivel de respuesta por su parte, a pesar del feddback que le proporciona el tribunal en el momento de la defensa. Esto nos lleva a pensar en la necesidad de ser más transparentes en la evaluación mediante la incorporación de mecanismos formales de feedback al alumno.

Finalmente, coincidimos con la opinión de Snavely y Wright (2003) quienes afirman que el alumno es responsable de su aprendizaje al poder elegir el tema, la orientación que se le da al trabajo y tener capacidad para profundizar más o menos en éste. Resultados acordes con los señalados por Rodríguez et al. (2008). Sin embargo, nuestra obligación como docentes es poner todos los medios a nuestro alcance para que finalmente se alcancen las competencias propuestas con la realización del Trabajo Fin de Grado.

Entre las limitaciones del estudio quisiéramos destacar el reducido tamaño de la muestra, aunque pensamos que los resultados ponen de manifiesto las percepciones del alumnado que merece la pena considerar en el transcurso de la mejora de los procesos de enseñanza-aprendizaje de cualquier institución educativa. Otra de las limitaciones podría ser el hecho de que se haya planteado en una sola titulación, pero creemos que como la metodología y el proceso de evaluación en este momento es común a cualquier plan de estudios, las conclusiones pueden ser válidas con pequeñas matizaciones por las características particulares de alguno de ellos.

\section{BIBLIOGRAFÍA}

alfaro tanco, J. A., Rodríguez Chacón, V. R., y AmORRortu gervasio, I. (2014): Desarrollo de competencias y habilidades a través de proyectos basados en empresas reales: Análisis en asignaturas de Dirección de Operaciones. Educade: Revista de Educación en Contabilidad, Finanzas y Administración de Empresas, 5, pp. 19-31.

BONILLA, M.J.; FUENTES, L.; VACAS, C.; VACAS, T. (2012): Análisis del proceso de evaluación del Trabajo Fin de Grado en las nuevas titulaciones, Educade. Revista de Educación en Contabilidad, Finanzas y Administración de Empresas, 3, pp. 5-21. 
Mercedes Ruíz Lozano, Pilar Tirado Valencia

La percepción de los alumnos sobre la adquisición de competencias del Trabajo Fin de Grado en ADE. Un análisis en la Universidad Loyola Andalucía

COLl, C; PAlacios, J. y MARChESI, A. (2008): Desarrollo Psicológico y Educación II. Psicología de la Educación. Madrid. Alianza Editorial.

COMISIÓN DE LAS COMUNIDADES EUROPEAS (2001): Making a European Area of Lifelong Learning a Reality. Bruselas, 21-11-2001 COM (2001) 678 final. Recuperado de http://aei.pitt.edu/42878/

Gallardo, G., y ReYES, P. (2010): Relación profesor-alumno en la universidad: arista fundamental para el aprendizaje. Calidad en la Educación, (32), pp. 77-108.

Hernández-leo, D., Moreno Oliver, V., Camps, I., Clarisó, R., Martinez-Monés, A., MarcoGALINDO, MaJ. Y MELERO, J. (2013): Implementación de buenas prácticas en los trabajos fin de grado. REDU, 11, pp. 269-278. Recuperado de http://redu.net/redu/index.php/REDU/article/view/652

ION, G. Y CANO, E. (2012): La formación del profesorado para la implementación de la evaluación por competencias. Educación XX1, 15(2), pp. 249-270. DOI: 10.5944/educxx1.15.2.141

Mateo, J.; Escofet, A.; Martínez Olmo, F. Ventura, J. y Vlachopoulos, D. (2012): Evaluating Tools in the European Higher Education Area (EHEA): an assessment for evaluating the competences of the Final Year Project in the Social Sciences. European Journal of Education, 47 (3), pp. 435-447. DOl:10.1111/j.14653435.2012.01536.x

Parlamento EuRopeo y CONSEjo, (2006): Recomendación 2006/962/CE del Parlamento Europeo y del Consejo, de 18 de diciembre de 2006, sobre las competencias clave para el aprendizaje permanente [Diario Oficial L 394 de 30.12.2006] Recuperado de http://eur-lex.europa.eu/legalcontent/ES/TXT/? uri=uriserv:c1 1090.

Robledo, P.; Fidalgo, R.; Arias, O. y Álvarez, M. L. (2015): Percepción de los estudiantes sobre el desarrollo de competencias a través de diferentes metodologías activas. Revista de Investigación Educativa, 33(2), pp. 369-383. DOl:http://dx.doi.org/10.6018/rie.33.2.201381

RodríGUeZ, J.M.; Alonso, M.; RUBIO, L. y EstebAN, C. (2008): Aprendizaje de competencias. Un análisis empírico en los estudios universitarios de Turismo en España. Calidad en la Educación, 28, pp. 278-304.

ROTHWELL, A. Y ARNOLD, J. (2007): Self-perceived employability: development and validation of a scale. Personnel Review, 36(1), pp. 23-41. DOI:http://dx.doi.org/10.1 108/00483480710716704

Rubio Hurtado, M J.; Ruiz Bueno, A. y Martínez-Olmo, F. (2016): Percepción del alumnado sobre la utilidad de las actividades de aprendizaje para desarrollar competencias. Revista de Investigación Educativa, 34(1), pp. 221-240. Recuperado de http://revistas.um.es/rie/article/view/225131 
Mercedes Ruíz Lozano, Pilar Tirado Valencia

La percepción de los alumnos sobre la adquisición de competencias del Trabajo Fin de Grado en ADE. Un análisis en la Universidad Loyola Andalucía

SNAVELY, L. L. Y WRIGHT, C. A. (2003): Research portfolio use in undergraduate honors education: assessment tool and model for future work. The Journal of Academic Librarianship, 29 (5), pp. 298-303. DOI: 10.1016/S0099-1333(03)00069-7

TejadA Fernández, J. y Ruiz Bueno, C. (2016): Evaluación de competencias profesionales en Educación Superior: Retos e implicaciones. Educación XX1, 19(1), pp. 17-38. DOI:10.5944/educXX1.12175

TODD, M. J., SMITH, K. Y BANNISTER, P. (2006): Supervising a social science undergraduate dissertation: staff experiences and perceptions. Teaching in Higher Education, 11 (2), pp. 161-173. DOI:10.1080/13562510500527693

TORRES-CORONAS, T. T. Y VIDAL-BLASCO, M. A. V. (2015): Percepción de estudiantes y empleadores sobre el desarrollo de competencias digitales en la educación superior. Revista de Educación, (367), pp. 63-90. DOI: 10.4438/1988-592X-RE2015-367-283

UnIVERSIDAD LOYOLA ANDALUCía (ULA), (2014a): Guía docente del Trabajo Fin de Grado del Grado en Administración y Dirección de Empresas. Curso 2014-2015. Recuperado de https://moodle.edu.uloyola.es/course/view.php?id=148

UnIVERSIDAD LOYOla ANDAluCía (ULA), (2014b): Normativa del Trabajo Fin de Grado para los estudios de Grado de la Universidad Loyola Andalucía. Curso 2014-2015. Recuperado de https://moodle.edu.uloyola.es/course/view.php?id=148

UnIVERSIDAD LOYOLA ANDALUCÍA (ULA), (2014c): Guía orientativa para los Trabajos Fin de Grado. Curso 2014-2015. Vicerrectorado de ordenación académica. Recuperado de https://moodle.edu.uloyola.es/course/view.php?id=148

Valderrama, E., Rullán, M., SÁnchez, F., PONS, J., Cores, F. y Bisbal, J. (2010): La evaluación de competencias en los Trabajos Fin de Grado. IEE-RITA, 5 (3), pp. 107-114. Recuperado de http://capinfo.e.ac.upc.edu/PDFs/dir10/file003867.pdf.

VILLA, A. y POBLETE, M. (2007): Aprendizaje basado en Competencias. Bilbao: Mensajero, Universidad de Deusto.

Wiggins, G. (1990): The Case for Authentic Assessment. Practical Assessment Research \& Evaluation, 2(2). Recuperado de http://pareonline.net/getvn.asp? v=2\&n=2. 